\title{
Inertia Force Balance of Crank-Group Driving Mechanism Based on Crank Unit
}

\author{
Yansong Liu*, Jujiang Cao, Sheng Ren and Yao Wu
}

College of Mechanical and Electrical Engineering, Shaanxi University of Science and Technology, Xi'an 710021, China

\begin{abstract}
The crank unit is defined as the basic unit of the crank-group driving mechanism for the modular design and production, and the balance methods of inertia force are researched on the crank unit in crank-group driving mechanism's modular design. The factors affecting the balancing weight's position and mass are studied. This indicates that the product of the mass and gyration radius of the balancing weight is influenced by the number, mass, length of the crank and the total mass moving rod in the mechanism. The balance method based on the crank unit is received to meet the modular design and production. This approach achieves the balance of crank-group driving mechanism quickly and efficiently.
\end{abstract}

Keywords: Crank-group driving mechanism, Crank unit, Inertia force balance, Modular design.

\section{INTRODUCTION}

The Crank-Group Driving Mechanism is a kind of planar linkages mechanism to realize multi-parallel shafts which rotate synchronously with redundant constraints. It is also suitable for the occasion of long center distance and small torque, such as tobacco machinery, agricultural products processing equipment (e.g. positioning mechanism of jujube slicing machine), packaging machinery, printing machinery and some special drill press etc. [1,2]. In the Crank-Group Driving Mechanism, the length and the phase of each crank is the same, every two cranks of mechanism can be regarded as a parallel double crank mechanism [3]. The general form of Crank-Group Driving Mechanism is shown in Fig. (1). The imbalance of high-speed mechanism will reduce the precision and working reliability of mechanism, or even destroy the mechanism [4]. Therefore, studying the balance conditions of Crank-Group Mechanism is necessary which has a good practical significance to mechanism design process. In practical applications, it needs different number of cranks (the number of transmission shafts) and the length of connecting rods (the shaft center distance) owing to the different usage scenario for Crank-Group Driving Mechanism. This also makes the number and the layout of crank's mechanism very different [5]. In order to simplify the problem and the mechanism's modular design, the Crank-Group Driving Mechanism can be divided into independent units with similar structure, namely the crank unit. The crank unit that comprises a crank and a connecting rod can be combined into different forms of Crank-Group Driving Mechanism to meet the requirements of different situations.

Changing the distribution of component's mass is one of the important methods to realize the inertia force balance of mechanism, and it is a fundamental problem that how to

*Address correspondence to this author at the College of Mechanical and Electrical Engineering, Shaanxi University of Science and Technology, Xi'an 710021, China; Tel: +8602986168806; E-mail: 360322715@qq.com derive the equilibrium equation of balance inertia force completely in the research of dynamic equilibrium of mechanism. The method of balancing inertia force of reciprocating motion mechanism can be classified as linear independent vector method and mass substitution method [611]. In this paper, in order to meet the requirements of modular design and its movement characteristics of CrankGroup Driving Mechanism [12-14], the inertia force balance of mechanism is achieved by calculating the centroid position and mass moment of each equalizer using mass substitution method.

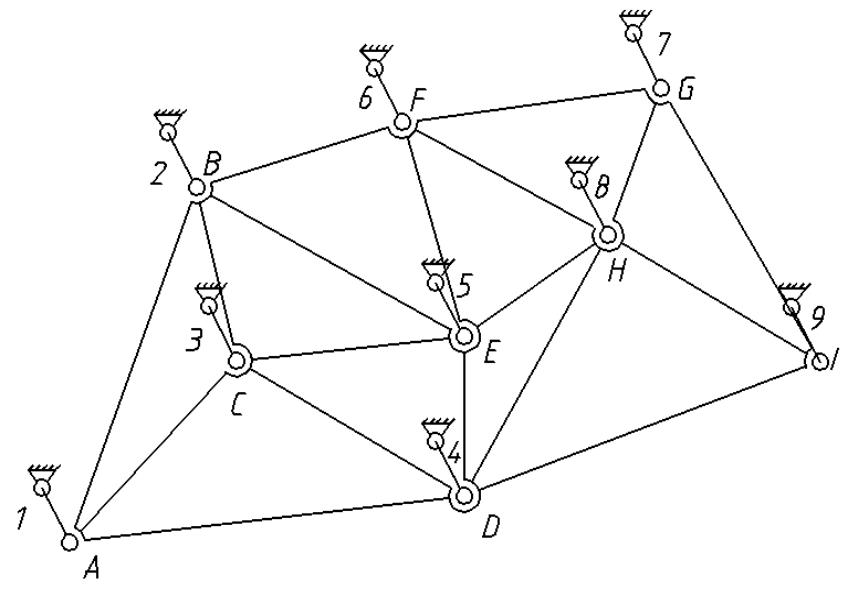

Fig. (1). General form of the crank-group driving mechanism.

\section{BALANCE OF CRANK-GROUP DRIVING MECHANISM}

For any Crank-Group Driving Mechanism, the coordinate system is set up as shown in Fig. (2). In order to realize the modular design and simplify structure of mechanism, it is assumed that the balancing weights have the same mass $m$ and gyration radius of centroid $L$, and the angle between the crank and the connection of centroid and the rotary center of the crank is $\theta_{0}$. 
The angle between the cranks and the $\mathrm{X}$-axis positive direction is $\theta$, and the positive direction of $\theta$ is the counterclockwise direction, so the angle between the $\mathrm{X}$-axis positive direction and the connection of balancing weight's centroid and the rotary center of crank is $\theta+\theta_{0}$. Assuming that all the rods of Crank-Group Driving Mechanism are homogeneous rods, the centroid of each rod is located at its center, the mass of each crank is $m_{0}$, the length of each crank is $r_{0}$, the number of cranks is $n$, the mass of connecting $\operatorname{rod} i$ is $m_{i}$, and the distance between rotary center of crank $k$ and the origin of coordinate is $S_{k}$. The angle between the X-axis positive direction and $S_{k}$ is $\theta_{k}$.

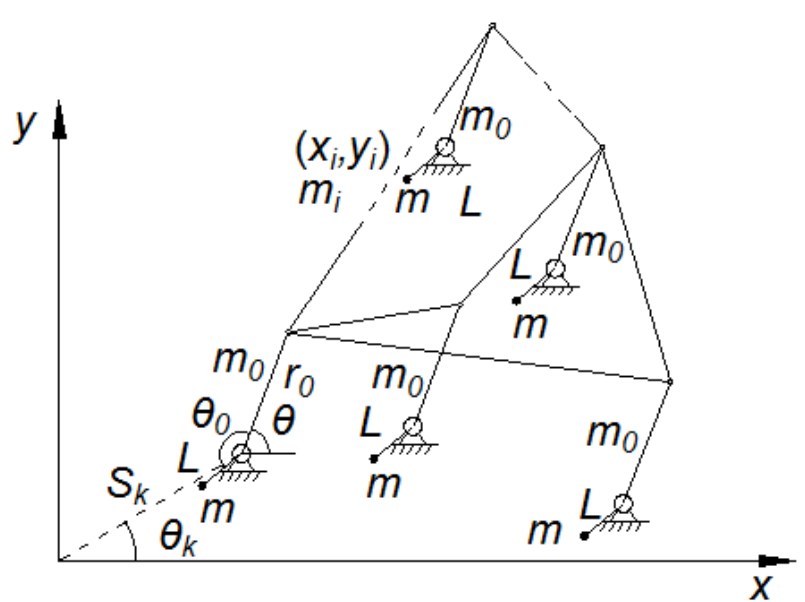

Fig. (2). Crank-group driving mechanism with $\mathrm{n}$ cranks.

According to the balance theory of mechanism, there are two methods to make the total centroid acceleration of the mechanism zero in order to achieve the inertia force balance of the mechanism. The first is that the total centroid of mechanism moves uniformly in a straight line, and the second is that the total centroid is stationary. The movement of Crank-Group Driving Mechanism is periodic, and the trajectory of the total centroid of mechanism is a closed curve, so the inertia force balance conditions of CrankGroup Driving Mechanism is that the total centroid of mechanism is stationary [15-17]. The mechanism must satisfy the following formula:

$\left\{\begin{array}{l}X_{s}=\frac{1}{M} \sum_{j=1}^{g} m_{j} x_{j}=C_{1} \\ Y_{s}=\frac{1}{M} \sum_{j=1}^{g} m_{j} y_{j}=C_{2}\end{array}\right.$

In formula (1), $\left(X_{S}, Y_{S}\right)$ represents the total centroid coordinates of the mechanism, $M$ is the total mass of the mechanism, $m_{j}$ is the mass of moving part $j,\left(x_{j}, y_{j}\right)$ represents the centroid coordinates of component $j, g$ is the number of moving members, and $C_{1}, C_{2}$ are two constants.

Formula (2) represents the coordinates of centroid of crank $k$.
$\left\{\begin{array}{l}X_{q k}=S_{k} \cos \theta_{k}+\frac{1}{2} r_{0} \cos \theta \\ Y_{q k}=S_{k} \sin \theta_{k}+\frac{1}{2} r_{0} \sin \theta\end{array}\right.$

Formula (3) indicates the coordinates of centroid of balancing weight $k$.

$\left\{\begin{array}{l}X_{p k}=S_{k} \cos \theta_{k}+L \cos \left(\theta+\theta_{0}\right) \\ Y_{p k}=S_{k} \sin \theta_{k}+L \sin \left(\theta+\theta_{0}\right)\end{array}\right.$

Formula (4) describes the centroid coordinates of the rod connecting the crank $k$ and the crank $h$.

$\left\{\begin{array}{l}X_{l k}=\frac{1}{2} S_{h} \cos \theta_{h}+\frac{1}{2} S_{k} \cos \theta_{k}+r_{0} \cos \theta \\ Y_{l k}=\frac{1}{2} S_{h} \sin \theta_{h}+\frac{1}{2} S_{k} \sin \theta_{k}+r_{0} \sin \theta\end{array}\right.$

For the Crank-Group Driving Mechanism determined form, only the values of the items with $\theta$ are variable in the centroid coordinates equation while others are constant. For making the value of centroid coordinates to become constant, the coefficient of those items with $\theta$ must be zero in order to satisfy the formula (1). The formula (5) is obtained by considering formulas (1 4).

$\left\{\begin{array}{l}n m L \cos \theta_{0}+\frac{1}{2} n m_{0} r_{0}+\sum m_{i} r_{0}=0 \\ n m L \sin \theta_{0}=0\end{array}\right.$

In the formula (5), $\sum m_{i}$ is the total mass of all the connecting rods.

The formula (6) is calculated by solving formula (5).

$\left\{\begin{array}{l}m L=\frac{1}{2} m_{0} r_{0}+\frac{1}{2} \sum m_{i} r_{0}=0 \\ \theta_{0}=180^{\circ}\end{array}\right.$

\section{INFLUENCE FACTORS OF INERTIA FORCE BALANCE OF CRANK-GROUP DRIVING MECHANISM}

The mass, the rotary radius and the distribution location of the balancing weight are determined by formula (6) for inertia force balance of the Crank-Group Driving Mechanism. In formula (6), $m_{0} r_{0} / 2$ is called crank item, and $\left(\sum m_{i} r_{0}\right) / n$ is called connecting rod item. Obviously, when each balancing weight is distributed on the opposite direction of the crank, the product of the mass and rotary radius of each balancing weight is influenced by the mass ( $\left.m_{0}\right)$ and the length $\left(r_{0}\right)$ of each crank, the number $(n)$ of the cranks, and the total mass $\left(\sum m_{i}\right)$ of the connecting rods. Formula (6) shows that it can achieve inertia force balance of the Crank-Group Driving Mechanism to add the same balancing weight to each crank. 
For different forms of Crank-Group Driving Mechanism with any number of cranks, the method of determining the inertial force balance parameters of the mechanism can be derived by formula (6):

(1) When a rank unit is added to the mechanism, the crank item $\left(m_{0} r_{0} / 2\right)$ remains unchanged, and the number of the cranks become from $n$ to $n+1$. The mass moment $(m L)$ of the balancing weight can be calculated by formula (6).

(2) The balancing weight is distributed in the opposite direction of the crank in each crank unit. The mass $(m)$ and rotary radius $(L)$ of the balancing weight may be determined according to the actual structure of the Crank-Group Driving Mechanism.

If the crank's center distance can only be selected in some given numbers, then mass value of connecting rod and the mass moment $(m L)$ of the balancing weight will only be in series numbers. So, the modular production of the CrankGroup Driving Mechanism can be achieved.

\section{EXAMPLE}

The Crank-Group Driving Mechanism with 3 cranks is shown in Fig. (3) and its parameters can be seen in Table 1.

Fig. (4) shows the total centroid coordinate curves of the mechanism without balancing weight. It means that the total centroid changes when the mechanism is moving.

According to the formula (6), the mass moment $m L$ of the balancing weight is 0.15 . The parameters of the balancing weight are set by $m=0.3 m, L=0.5 m, \theta_{0}=180^{\circ}$. After adding the balancing weights to the mechanism, the total centroid coordinates of the mechanism are constant as shown in Fig. (5). It validates that the inertia force balance of the Crank-Group Driving Mechanism is achieved.

Table 1. Parameters of Crank-Group Driving Mechanism.

\begin{tabular}{|c|c|c|c|c|c|}
\hline $\mathbf{m}_{\mathbf{0}} / \mathbf{k g}$ & $\mathbf{m}_{\mathbf{1}} / \mathbf{k g}$ & $\mathbf{m}_{\mathbf{2}} / \mathbf{k g}$ & $\mathbf{m}_{\mathbf{2}} / \mathbf{k g}$ & $\mathbf{s}_{\mathbf{1}} / \mathbf{m}$ & $\mathbf{s}_{2} / \mathbf{m}$ \\
\hline \hline 0.2 & 0.3 & 0.4 & 0.5 & 0.141 & 0.728 \\
\hline $\mathrm{s}_{3} / \mathrm{m}$ & $\mathrm{r}_{0} / \mathrm{m}$ & $\theta_{1} \rho$ & $\theta_{2} \rho$ & $\theta_{3} \rho$ & \\
\hline 1.581 & 0.3 & 45 & 16 & 35 & \\
\hline
\end{tabular}

\section{CONCLUSION}

(1) The crank unit has been defined in this paper, and the important role of the crank unit has been discussed in the process of modular design and production of the Crank-Group Driving Mechanism.

(2) A method achieving inertia force balancing of the Crank-Group Driving Mechanism has been proposed according to the special structure of the mechanism. The factors influencing mass moment of the balancing weights and its calculation method are elaborated. The study provides the theoretical basis for the modular design of the Crank-Group Driving Mechanism.

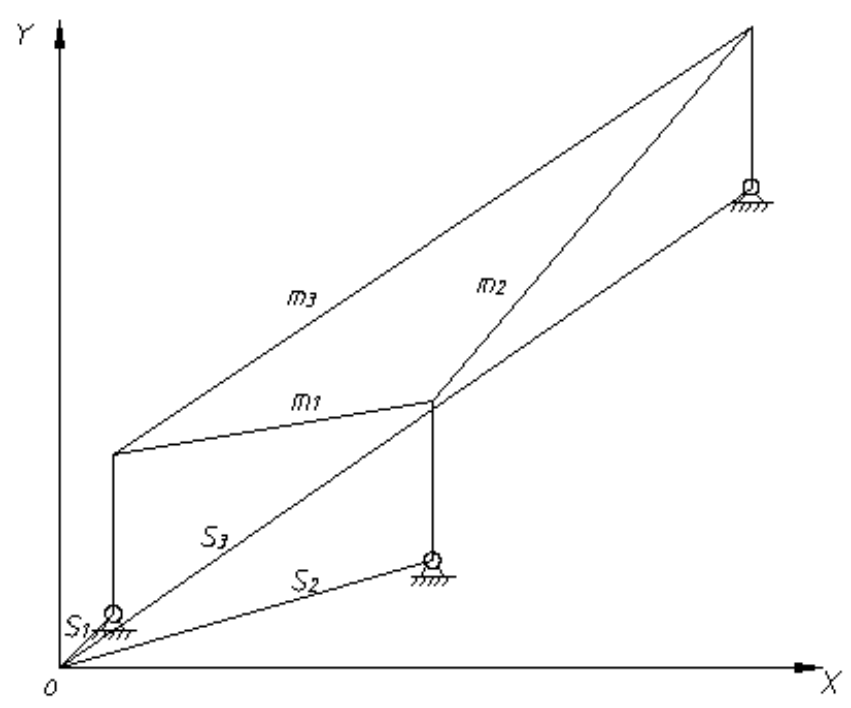

Fig. (3). Crank-Group Driving Mechanism with 3 cranks.

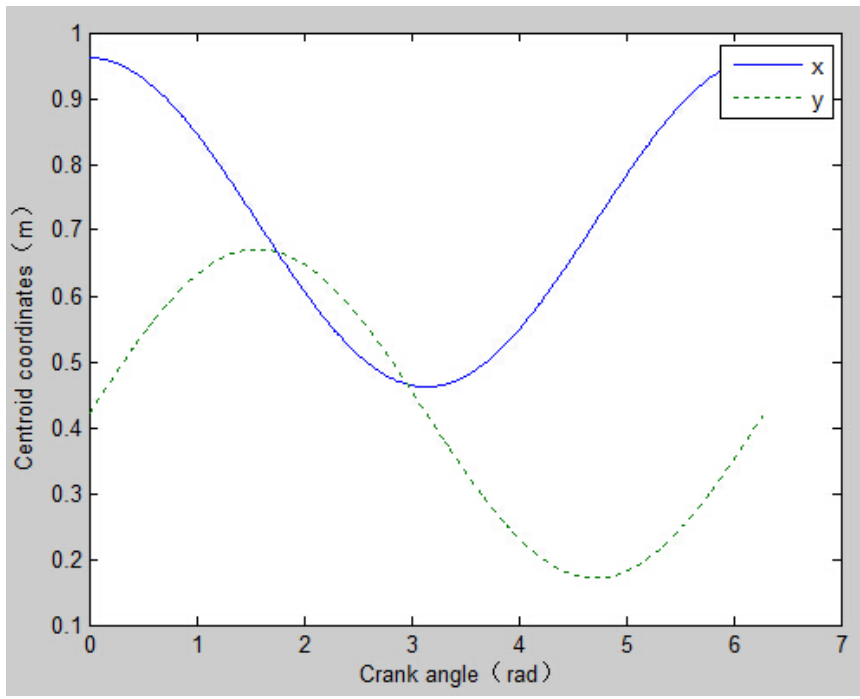

Fig. (4). The coordinate of total centroid of moving parts without balancing weight.

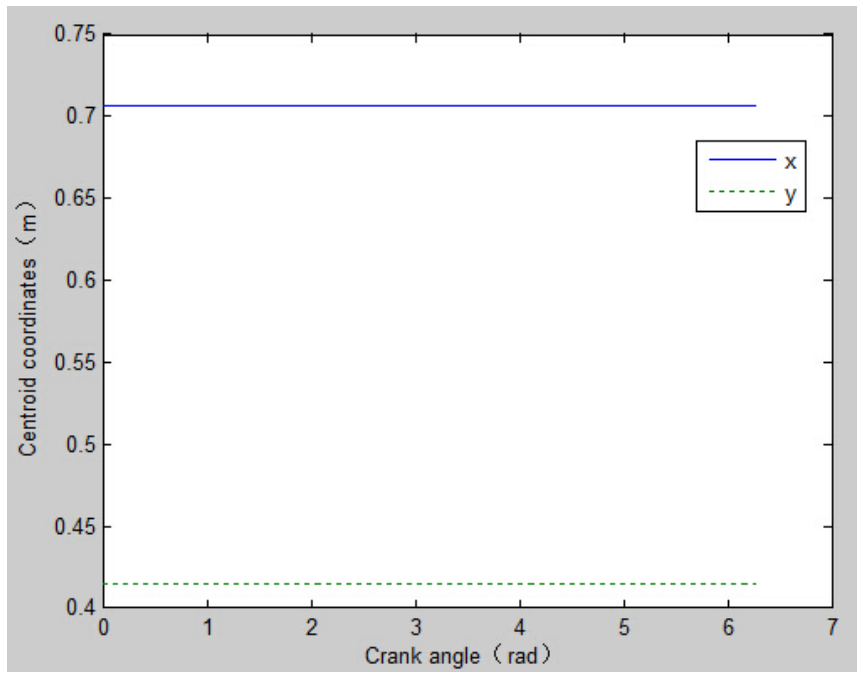

Fig. (5). Total centroid coordinate of moving parts with balancing weight. 


\section{CONFLICT OF INTEREST}

The authors confirm that this article content has no conflict of interest.

\section{ACKNOWLEDGEMENTS}

The research described in this paper was partially supported by NSFC (Serial number: 51175313), China.

\section{REFERENCES}

[1] L. O. Barton, "Mechanism analysis of a trisector," Mechanism and Machine Theory, vol. 43, no. 2, pp. 115-122, 2008.

[2] W. Lu, "Design and Application of Single-link and Multi-crank mechanism in Crank Driving for Multi-axle Box," Journal of Changzhou Industrial Technology College (Natural Science Edition), vol. 9, no. 2, pp. 46-51, 1996.

[3] Y. Wang, and J. Cao, "The Overview of Crank-group Driving Mechanism," Journal of Mechanical Transmission, vol. 37, no. 4, pp. 134-136, 2013.

[4] W. Wang, J. Cao, and Y. Liu, "Study on the dynamic balance of crank unit in crank-group driving mechanism," Journal of Mechanical Transmission, vol. 38, no. 8, pp. 23-25, 2014.

[5] Z. Liu, and Z. Feng, "Theoretical Study of Integral Balancing of First-order Reciprocal Inertia of Small-sized Single-cylinder Horizontal Engine," Journal of Agricultural Machinery, no. 3, pp. 23-28, 1990.

[6] R. Guo, and X. Kong, "A mass moment substitution approach to shaking force complete balancing of planar linkages," Journal of Mechanical Transmission, vol. 43, no. 1, pp. 9-12, 2000.
[7] F. Gao, and Y. Zhao, "Complete shaking force balancing of multiloop spatial by using unit vector method," Journal of Northeast Heavy Machinery Institute, vol. 16, no. 2, pp. 127-133, 1992.

[8] M. L. Walker, and K.A. Oldham, "General Theory of Force Balancing Using Counterweights," Mechanisms and Machine Theory, vol. 13, no. 3, pp. 175-183, 1978 .

[9] X. Kong, and L. Yang, "A Mass Moment Substitution Method for Complete Shaking Force Balancing of Spatial Linkages Involving $\mathrm{R}$ and P Pairs Part 1-Mass Moment Substitution," Mechanical Science and Technology, vol. 16, no. 4, pp. 575-580, 1997.

[10] Y. Yang, and D. Jin, "Mechanical System Dynamics," Tsinghua University Press, Beijing: 2009.

[11] B. Liao, Y. Luo, Z. Shi, B. Zhang, and Y. Hu, "Application of place method for balancing shaking in spatial mechanisms," Journal of Nachang University (Engineering \& Technology), vol. 34, no. 2, pp. 176-178, 2012.

[12] N. Jiao, L. Zhang, and Z. Lu, "Unitized design and motion simulation of crank group driving mechanism," Journal of Mechanical Transmission, vol. 38, no. 2, pp. 86-89, 2014.

[13] Y. Wang, and J. Cao, "Study on the motion feature of crank- group driving mechanism," Journal of Mechanical Transmission, vol. 37, no. 10 , pp. 79-81, 2013.

[14] S. Ren, J. Cao, Y. Liu, "Kinematic analysis of crank-group driving mechanism based on influence coefficient method," Journal of Mechanical Transmission, vol. 38, no. 8, pp. 104-106, 2014.

[15] Y. Yu, and Z. Li, "Modern mechanical dynamics," Beijing Industrial University Press, Beijing: 1998.

[16] C. Zhang, "Mechanical dynamics," Higher Education Press, Beijing: 2008.

[17] Z. Mei, R. Yang, B. Zhou, and G. Zhang, "On the balancing of planar 3 DOF parallel manipulator," Mechanical Science and Technology, vol. 22, no. 6, pp. 968-970, 2003. 